\title{
An ad libitum meal provided with or without fluid and either euhydrated or hypohydrated does not affect food intake
}

\author{
Robert A. Corney ${ }^{1}$, Caroline Sunderland ${ }^{2}$ and Lewis J. James ${ }^{1}$ \\ ${ }^{1}$ SSEHS, Loughborough University and ${ }^{2} S S T$, Nottingham Trent University, UK
}

Research has shown that restriction of fluid intake reduces food intake in animals ${ }^{(1)}$ and humans ${ }^{(2)}$. Shirreffs et al. ${ }^{(2)}$ reported a $29 \%$ reduction in energy intake when subjects completely restricted fluid intake for $37 \mathrm{~h}$, although food choices were limited to dry foods only during fluid restriction. In contrast, we have previously reported ${ }^{(3)}$ that hypohydration of $\sim 2.7 \%$ does not affect energy intake at a buffet meal compared to a euhydrated trial when fluids are provided with the meal. Taken together, these results suggest that fluid restriction and not hypohydration reduces food intake or that the provision of dry foods during fluid restriction might account for the previous findings. Thus the purpose of the present study was to examine the interaction between hypohydration and fluid availability on voluntary energy intake.

16 healthy males (age: 25 (4) y, body mass: $72.6(8.6) \mathrm{kg}$, height: $1.78(0.07) \mathrm{m})$ completed four $24 \mathrm{~h}$ trials in randomised, counterbalanced order: euhydrated with fluid provided at the ad libitum meal (EU-F), euhydrated with no fluid provided at the ad libitum meal (EU-NF), hypohydrated with fluid provided at the ad libitum meal (HYPO-F), hypohydrated with no fluid provided at the ad libitum meal (HYPO-NF). For all trials, food and water $\left(40 . \mathrm{ml}_{\mathrm{kg}}{ }^{-1}\right.$ body mass; EU trials only) was provided to subjects. Subjects visited the laboratory overnight fasted on two consecutive mornings ( $0 \mathrm{~h}$ and $24 \mathrm{~h})$. Body mass was measured, urine and blood samples provided and a subjective feelings questionnaire completed at $0 \mathrm{~h}$ and $24 \mathrm{~h}$. At $24 \mathrm{~h}$, subjects consumed an ad libitum porridge breakfast and ate to satiety for $30 \mathrm{~min}$.

Body mass decreased during HYPO-F $(-1.74(0.39) \%)$ and HYPO-NF $(-1.89(0.54) \%)$ trials $(P<0.001)$, but did not change during EU-F $(-0.17(0.68) \%)$ of EU-NF $(-0.35(0.40) \%)$ trials $(P>0.05)$. Total urine volume was greater during EU-F $(2262(494) \mathrm{ml})$ and EU-NF (2477 (494) ml) than during HYPO-F (724 (272) ml) and HYPO-NF (806 (201) ml) $(P<0.001)$. Compared to 0 h, serum osmolality increased and plasma volume decreased during HYPO-F and HYPO-NF $(P<0.001)$, but did not change during EU-F and EU-NF trials $(P>0.05)$. Energy intake at the ad libitum meal was 2693 (938) kJ (EU-F), 2367 (643) kJ (EU-NF), 2268 (529) kJ (HYPO-F), 2396 (954) kJ (HYPO-NF) and was not different between trials $(P=0.125)$. When fluid was provided with the ad libitum meal fluid intake was greater during HYPO-F $(626(258) \mathrm{ml})$ than EU-F (407 (254) ml) $(P<0.01)$. With the exception of thirst, there were no main effects of trial or interaction for any other subjective feelings.

These results demonstrate that in a laboratory setting there appears to be little effect of hypohydration or fluid availability during feeding on energy intake at an ad libitum breakfast when subjects are provided with a typical semi solid breakfast meal. This suggests that the results of previous studies might therefore be related to the provision of dry foods during fluid restriction.

1. Watts AG \& Boyle CN (2010) The functional architecture of dehydration-anorexia. Physiol Behav 100, 472-477.

2. Shirreffs SM, Merson SJ, Fraser SM et al. (2004) The effects of fluid restriction on hydration status and subjective feelings in man. $B r J$ Nutr $\mathbf{9 1}$, 951-958.

3. Corney RA, Sunderland C \& James LJ (2013) The effect of rehydration after dehydrating exercise on appetite and energy intake. Int J Sport Nutr Exerc Metab 23, S9. 